\title{
Killian-Jamieson diverticulum mimicking a calcified thyroid nodule on ultrasonography: A case report and literature review
}

\author{
$\mathrm{LI} \mathrm{CAO}^{1^{*}}, \mathrm{JUNNA} \mathrm{GE}^{2 *}$, DONGHUI ZHAO ${ }^{2}$ and SHANGTONG LEI ${ }^{2}$ \\ Departments of ${ }^{1}$ Breast Center and ${ }^{2}$ General Surgery, Nanfang Hospital, Southern Medical University, \\ Guangzhou, Guangdong 510515, P.R. China
}

Received April 11, 2015; Accepted July 1, 2016

DOI: $10.3892 / \mathrm{ol} .2016 .4984$

\begin{abstract}
As a rare hypopharyngeal diverticulum, Killian-Jamieson diverticulum (KJD) is usually incidentally detected, small, asymptomatic and likely to be misdiagnosed as a thyroid nodule. In order to avoid unnecessary treatments resulting from misdiagnosis, principles to distinguish between esophageal diverticula and thyroid nodules should be noted clearly. The current study presents a case of an asymptomatic unilateral KJD that mimicked a calcified thyroid nodule. In the current case, a 40-year-old man presented with a 'suspected malignant thyroid nodule' in the left thyroid gland, and underwent left thyroid lobectomy and a neck exploration. However, no visible 'suspected tumor', but a sac protruding from the left anterolateral wall of the cervical esophagus, was observed during the surgery. A swallow test on ultrasonography (US) in combination with pharyngoesophagography was then performed, which confirmed the diagnosis. The patient recovered well during the 3-month follow-up after the operation. The present study indicates that real-time US and pharyngoesophagography are important techniques to distinguish a KJD from a thyroid nodule so that unnecessary surgical intervention can be avoided.
\end{abstract}

\section{Introduction}

Ultrasonography (US) of the thyroid is a well-established method for evaluating thyroid nodules. However, occasionally, non-thyroidal lesions such as diverticula may be confused

Correspondence to: Professor Shangtong Lei or Professor Donghui Zhao, Department of General Surgery, Nanfang Hospital, Southern Medical University, 1838 North Guangzhou Avenue, Guangzhou, Guangdong 510515, P.R. China

E-mail: leishangtong2015 @126.com

E-mail: zhaodonghui2015@126.com

*Contributed equally

Key words: calcification, surgery, Killian-Jamieson esophageal diverticulum, complications with thyroid nodules on physical examination or imaging studies (1-3).

Pharyngoesophageal diverticula, including KillianJamieson diverticulum (KJD) and Zenker's diverticulum (ZD), are rare hypopharyngeal diverticula that could easily be misdiagnosed as thyroid nodules $(2,4,5)$. ZD was reported to be the most common diverticula of the esophagus, but the rate of KJD was only about a forth of that of ZD $(6,7)$. In a small number of patients, KJD and ZD can coexist (8). The two types of diverticula protrude through a muscular gap of anatomical weakness in the cervical esophagus near the cricopharyngeus muscle. KJD originates in the anterolateral wall just inferior to the cricopharyngeus muscle and superolateral to the longitudinal muscle of the esophagus (9), whereas ZD originates in the posterior wall above the cricopharyngeus, which protrudes posteriorly, and usually projects to the left (10). Few clinicians or sonographers are aware of the possibility of diverticula mimicking thyroid nodules.

The current study presents a case of an asymptomatic unilateral KJD that mimicked a calcified thyroid nodule, and reviews the literature of this uncommon presentation. This will enable clinicians to view another source of information on the subject and means that unnecessary invasive procedures such as surgery can be avoided.

\section{Case report}

A 40-year-old man presented with a suspected thyroid nodule that was identified incidentally on a routine checkup in a local hospital on November 10, 2014. Thyroid US demonstrated a hypoechoic nodule in the left thyroid lobe. The patient presented with no dysphagia, hoarseness, regurgitation, odynophagia, halitosis, chronic coughing, neck discomfort, gastroesophageal reflux symptoms or fever, and the medical and surgical histories were unremarkable. The patient was subsequently referred to Nanfang Hospital (Guangzhou, Guangdong, China) for further evaluation on December 14, 2014. The physical examination of the head and neck was unremarkable. In addition, laboratory data revealed no abnormalities. Serum triiodothyronine, thyroxine and thyroid-stimulating hormone concentrations were $0.88 \mathrm{ng} / \mathrm{mL}$ (normal range, $0.60-1.81 \mathrm{ng} / \mathrm{ml}$ ), $7.3 \mu \mathrm{g} / \mathrm{dl}$ (normal range, 4.5-10.9 $\mu \mathrm{g} / \mathrm{dl}$ ) and $2.22 \mathrm{mIU} / \mathrm{l}$ (normal range, 0.55-4.78 mIU/l), respectively, and no thyroid autoantibodies were detected. A further US examination was performed 


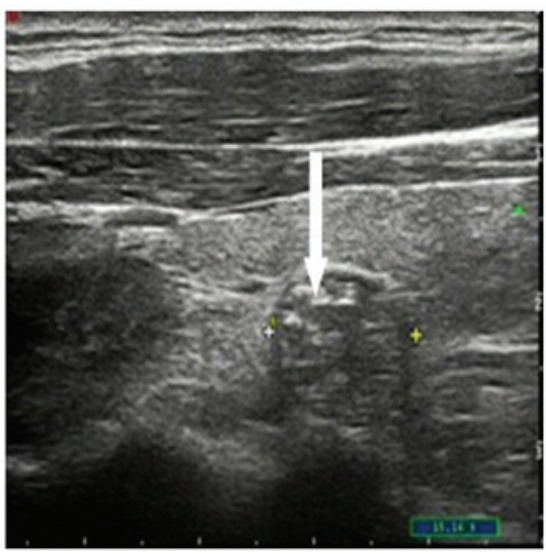

Figure 1. Transverse sonogram showing a $1.1 \times 1.0-\mathrm{cm}$ hypoechoic nodule (arrow) in the upper portion of the left thyroid lobe with internal hyperechoic foci (arrowhead) and a hypoechoic rim.

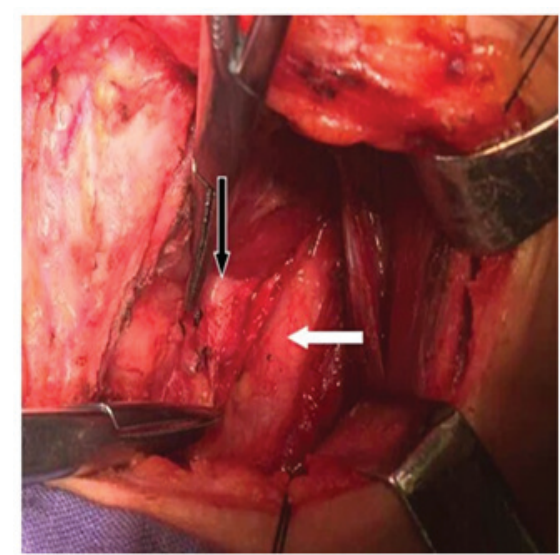

Figure 2. Intraoperative image obtained during the neck exploration after the left thyroid lobectomy showing a left-sided hypopharyngeal diverticulum sac protruding from the left anterolateral wall of the cervical esophagus, which is visible under the cricopharyngeus muscle. The black arrow indicates the diverticulum and the white arrow indicates the trachea.

following admission, which showed a $1.3 \times 1.0-\mathrm{cm}$ hypoechoic lesion containing internal-hyperechoic foci and a hypoechoic rim in the upper portion of the left thyroid gland, suggesting papillary carcinoma with punctate calcifications (Fig. 1). On power Doppler imaging, the lesion also showed internal vascularity. The rest of the thyroid gland was normal. No pathological lymphadenopathy was noted at the time. The patient reported a history of hypertension for 2 years that was not treated by medication, with the highest blood pressure reaching 140/90 mmHg (normal values, <120/80 $\mathrm{mmHg}$ ).

In order to remove the suspected malignant thyroid nodule, the patient underwent a left thyroid lobectomy and a neck exploration under general anesthesia on December 19, 2014, taking care not to injure the recurrent laryngeal nerve, which runs in the tracheoesophageal groove. However, when surgical cutdowns were performed for the removed left thyroid gland tissue, no visible suspected tumor was observed, only calcification. Histopathological examination revealed a (left thyroid) nodular goiter with focal calcifications. During the further exploration, a sac was found protruding from the left anterolateral wall of the cervical esophagus, which is visible under the
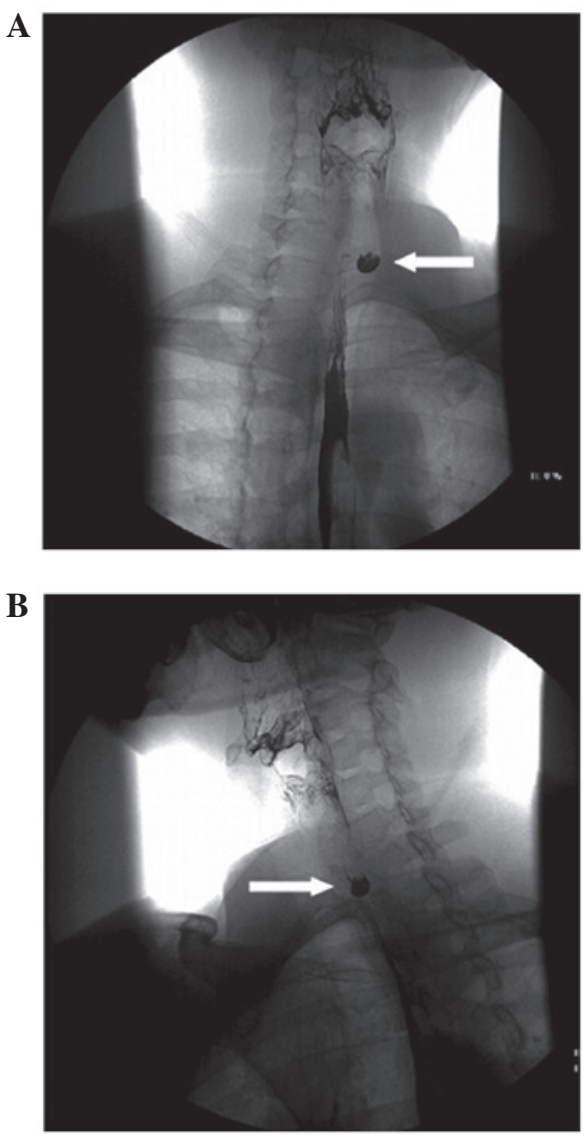

Figure 3. Pharyngoesophagography on day 3 after the left thyroid lobectomy on (A) anteroposterior and (B) lateral projections showing a sac-like outpouching structure (white arrow) arising from the anterolateral wall of the cervical esophagus, suggesting a left Killian-Jamieson diverticulum.

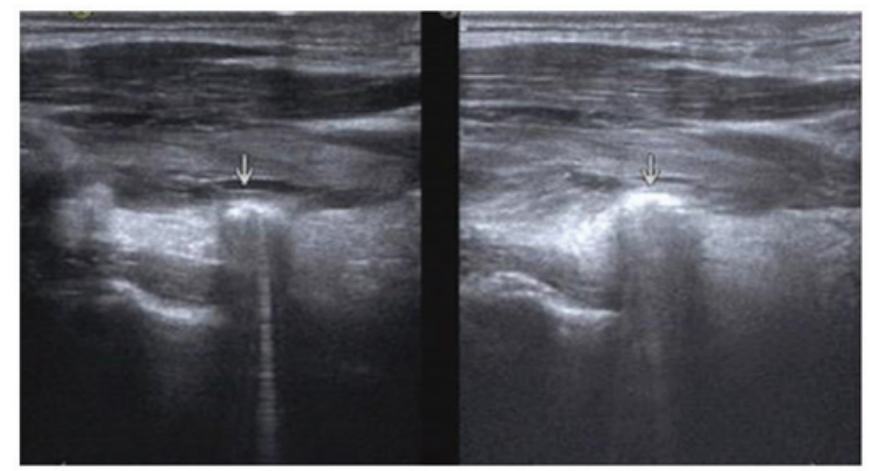

Figure 4. Transverse sonogram obtained on repeat ultrasonography examination on day 4 after the left thyroid lobectomy showing the connection between the nodule and the esophageal wall. In the swallow test, changes in the hyperechoic lesions and internal echogenic foci were observed to be consistent with air and water moving into the esophagus.

cricopharyngeus muscle (Fig. 2). The sac was located $\sim 5 \mathrm{~mm}$ lateral to the point where the recurrent laryngeal nerve enters the pharynx. Considering the diverticulum was asymptomatic and small, further surgery was not performed. The wound was closed in layers with placement of a closed suction drain. The drain was removed on post-operative day 2 . On post-operative day 3 , subsequent upper gastrointestinal radiography on the anteroposterior (Fig. 3A) and lateral (Fig. 3B) projections 
Table I. Clinical and ultrasonographic findings of Killian-Jamieson diverticulum presenting as a thyroid nodule reported in the literature.

\begin{tabular}{|c|c|c|c|c|c|c|}
\hline $\begin{array}{l}\text { First author, } \\
\text { year }\end{array}$ & Age/gender & Chief complaint & Size, $\mathrm{cm}$ & Side & $\begin{array}{c}\text { Surgical } \\
\text { intervention }\end{array}$ & (Ref.) \\
\hline Boisvert et al, 2010 & $69 / \mathrm{M}$ & $\begin{array}{l}\text { Progressive dysphagia, } \\
\text { nighttime coughing, } \\
\text { hoarseness and } \\
\text { gastroesophageal reflux }\end{array}$ & $\begin{array}{l}\text { Left: } 3.5 \\
\text { Right: } 4.4\end{array}$ & Bilaterally & Yes & (9) \\
\hline \multirow[t]{4}{*}{ Kim et al, 2012} & $55 / \mathrm{F}$ & $\begin{array}{l}\text { Increased size of known } \\
\text { thyroid nodule }\end{array}$ & 4.0 & Left & Yes & $(10)$ \\
\hline & $50 / \mathrm{M}$ & Incidentally detected & 1.2 & Left & Yes & \\
\hline & $55 / \mathrm{M}$ & Incidentally detected & 1.2 & Left & No & \\
\hline & $59 / \mathrm{F}$ & Incidentally detected & 1.7 & Left & No & \\
\hline Tang et al, 2008 & $51 / \mathrm{F}$ & $\begin{array}{l}\text { Dysphagia, a globus sensation } \\
\text { posterior to the sternum }\end{array}$ & 1.5 & Left & Yes & (14) \\
\hline Kim et al, 2012 & $68 / \mathrm{M}$ & Epigastric pain & 10.0 & Right & Yes & $(15)$ \\
\hline Mimatsu et al, 2013 & $77 / \mathrm{F}$ & Mild dysphagia & $\begin{array}{l}\text { Left: } 4.0 \\
\text { Right: } 1.5\end{array}$ & Bilaterally & Yes & (16) \\
\hline Pang et al, 2009 & $54 / \mathrm{M}$ & Incidentally detected & 1.1 & Left & Yes & $(17)$ \\
\hline Present study & $40 / \mathrm{M}$ & Incidentally detected & 1.3 & Left & No & \\
\hline
\end{tabular}

F, female; M, male

demonstrated a $1.3 \times 1.0-\mathrm{cm}$ KJD originating from the left wall of the esophagus (C6-C7 level). To confirm the diagnosis of an esophageal diverticulum, the patient was administered a swallow test during US on post-operative day 4 . When the patient swallowed, multiple hyperechoic spots were observed (Fig. 4). Real-time US was performed showing that the movements of the hyperechoic spots were consistent with air and water moving into the esophagus. Transient changes were also demonstrated, including an increase in the size of the lesion, a reduction in the definition of the margins and heterogeneous echogenicity of the contents of the lesion. The patient recovered without complications such as nerve damage or hemorrhage, and was then discharged from hospital. During the 3-month follow-up observations, the patient did not show any abnormalities, such as diverticular relapse, dysphagia or stenosis.

\section{Discussion}

KJD is an outpouching from the lateral wall of the proximal cervical esophagus. KJD protrudes through a muscular gap called the KJ triangle, which was first described by Killian and then confirmed by Jamieson $(11,12)$. The muscular gap is in the anterolateral wall of the proximal esophagus, inferior to the cricopharyngeus muscle and superolateral to the longitudinal muscle of the esophagus (11-13).

The pathogenesis of KJD is unclear. It is hypothesized that the KJD is the result of a functional outflow obstruction in the esophagus, the anatomical muscle weakness of the $\mathrm{KJ}$ space, high intraluminal pressure building against a weakness in the gastrointestinal tract wall or food residue accumulation within the diverticulum, with possible non-specific chronic inflammation within and around the diverticulum resulting in esophageal dysmotility $(10,14)$.
Previously reported cases of KJD mimicking thyroid nodules were reviewed in the present study to determine the clinical and US characteristics of KJD $(9,10,14-17)$. The following terms were searched in the title and/or abstract of articles published in the PubMed database on December 26, 2014: 'Killian-Jamieson diverticula', 'Killian-Jamieson diverticulum' or 'KJD'. Ultimately, 10 cases of KJD, including the present case, were analyzed (Table I). Of the 10 patients reviewed, 6 patients were men and 4 were women. The mean age of all 10 patients was 57.8 years, with a range of 40-77 years. In total, $12 \mathrm{KJD}$ were detected on pharyngoesophagograms in 10 patients, including $7(70 \%)$ with unilateral left-sided diverticula, $1(10 \%)$ with right-sided diverticula and $2(20 \%)$ with bilateral diverticula. Additionally, 6 out of the 10 patients exhibited no symptoms, with the exception of 1 patient, who exhibited a neck mass. The remaining 4 patients exhibited symptoms such as dysphagia, coughing, hoarseness, gastroesophageal reflux and a globus sensation. The mean maximal dimension of the diverticula was $2.95 \mathrm{~cm}$, ranging from $1.1-10 \mathrm{~cm}$. A total of 7 patients were treated with surgical intervention, including fine-needle aspiration (FNA), open surgical excision of the diverticulum or endoscopic diverticulotomy.

Unlike the other pharyngoesophageal diverticulum, ZD, which is more common and symptomatic, KJD is smaller and more likely to be asymptomatic, resulting in its increased chance to be misdiagnosed as a thyroid nodule (5). As in the present study, the study by Rubesin and Levine (8) found KJD to be asymptomatic in the majority of patients $(89 \%)$. Dysphagia, coughing and epigastric pain are the most common symptoms experienced by patients with KJD (1). Retention of food material in the diverticulum may lead to regurgitation after meals and chronic coughing, and even to aspiration pneumonia, particularly in large diverticula (8). 
Esophageal diverticula are usually detected incidentally by an esophagography. On occasion, diverticula can be found by thyroid US when they mimic thyroid nodules (10). The diagnostic rate is closely associated with the doctor's working attitude and practical skill. Due to the existence of air bubbles and food material, diverticula can be imaged as heterogeneous internal echoes with strong echogenic foci, which strongly suggest microcalcifications of papillary thyroid cancer $(4,18)$. Thus, in order to avoid unnecessary treatment resulting from a misdiagnosis, principles to distinguish between esophageal diverticula and thyroid nodules should be noted clearly. Kim et al summarized the US characteristics of esophageal diverticula (10). The real-time US used in a swallow test may be of great assistance. When the patient was swallowing, the hyperechoic lesions and internal echogenic foci may change to be consistent with air and water moving into the esophagus, as in the present case (19). However, it should be mentioned that these changes and even the connection with the esophageal wall cannot be observed in all cases, suggesting a further barium swallow pharyngoesophagography is necessary when a diverticulum is considered $(20,21)$.

When a diverticulum masquerades as a thyroid nodule, FNA may be performed to obtain a pathological diagnosis (3). However, complications have not been recorded or reported in such cases. To the best of our knowledge, as the $\mathrm{KJ}$ triangle is directly adjacent to the entry point of the recurrent laryngeal nerve into the larynx, FNA or endoscopic treatment is quite dangerous (6). If patients have large diverticula or are symptomatic, they should be treated with surgical intervention. Therefore, it is important to note that careful dissection in this area is necessary to avoid nerve injury. We recommend that FNA or endoscopic treatment should be chosen cautiously. However, the traditional open surgical approach is currently being challenged by the use of endoscopic diverticulotomy (22). One of the potential explanations for this may be its advantage of intracavitary drainage over open surgical treatment. Time is required to prove which one technique is more efficient.

In conclusion, the present study reported the case of a man who presented with a 'suspected malignant thyroid nodule' in the left thyroid gland, and subsequently underwent left thyroid lobectomy and a neck exploration. During the surgery, the nodule was identified as a KJD. The present findings suggest that real-time US and pharyngoesophagography are important techniques to distinguish a KJD from a thyroid nodule, so that unnecessary surgical intervention can be avoided. Open surgical or endoscopic diverticulotomy is required when patients have large diverticula or are symptomatic, although the preference for either technique is controversial. However, considering that the recurrent laryngeal nerve crosses the KJ space, we suggest that FNA or endoscopic treatment should be chosen cautiously in order to avoid nerve injury.

\section{References}

1. DeFriend DE and Dubbins PA: Sonographic demonstration of a pharyngoesophageal diverticulum. J Clin Ultrasound 28: 485-487, 2000.

2. Hayashi N, Tamaki N, Konishi J, Endo K, Misaki T, Torizuka K and Mori T: Lateral pharyngoesophageal diverticulum simulating thyroid adenoma on sonography. J Clin Ultrasound 12: 592-594, 1984.

3. Seiberling KA, Dutra JC and Gunn J: Ultrasound-guided fine needle aspiration biopsy of thyroid nodules performed in the office. Laryngoscope 118: 228-231, 2008.

4. Kumar A, Aggarwal S and Pham DH: Pharyngoesophageal (Zenker's) diverticulum mimicking thyroid nodule on ultrasonography: Report of two cases. J Ultrasound Med 13: 319-322, 1994.

5. Mercer D, Blachar A, Khafif A, Weiss J and Kessler A: Real-time sonography of Killian-Jamieson diverticulum and its differentiation from thyroid nodules. J Ultrasound Med 24: 557-560, 2005.

6. Rekhtman N, Rekhtman K, Sheth S and Ali SZ: A 62-year-old woman with a suspected thyroid nodule. Arch Pathol Lab Med 129: 1497-1498, 2005.

7. Puricelli MD and Zitsch RP III: Is it really a thyroid nodule? Another cause of a lower midline neck mass. Otolaryngol Head Neck Surg 147: 397-398, 2012.

8. Rubesin SE and Levine MS: Killian-Jamieson diverticula: Radiographic findings in 16 patients. AJR Am J Roentgenol 177: 85-89, 2001.

9. Boisvert RD, Bethune DC, Acton D and Klassen DR: Bilateral Killian-Jamieson diverticula: A case report and literature review. Can J Gastroenterol 24: 173-174, 2010.

10. Kim HK, Lee JI, Jang HW, Bae SY, Lee JH, Kim YS, Shin JH, Kim SW and Chung JH: Characteristics of Killian-Jamieson diverticula mimicking a thyroid nodule. Head Neck 34: 599-603, 2012

11. Jamieson EB: Section II, Head and Neck, (64 plates), 15s. net. In: Illustrations of Regional Anatomy. 3rd edition. E. \& S. Livingstone, Edinburgh, p44, 1941.

12. Zanwar VG, Gambhire1 PA, Choksey AS and Rathi PM: KillianJamieson diverticulum: Cervical oesophageal diverticulum. Journal of the Association of Physicians of India 63: 65-66, 2015.

13. Zaino C, Jacobson HG, Lepow H and Ozturk C: The Pharyngoesophageal Sphincter. Charles C. Thomas, Publisher, Ltd., Springfield, IL, pp29-144, 1950.

14. Tang SJ, Tang L, Chen E and Myers LL: Flexible endoscopic Killian-Jamieson diverticulotomy and literature review (with video). Gastrointest Endosc 68: 790-793, 2008.

15. Kim DC, Hwang JJ, Lee WS, Lee SA, Kim YH and Chee HK: Surgical treatment of killian-jamieson diverticulum. Korean J Thorac Cardiovasc Surg 45: 272-274, 2012.

16. Mimatsu K, Oida T, Kano H, Kawasaki A, Fukino N, Kida K, Kuboi Y and Amano S: Killian-Jamieson diverticula presenting synchronously with thyroid adenoma. Case Rep Gastroenterol 7: 188-194, 2013.

17. Pang JC, Chong S, Na HI, Kim YS, Park SJ and Kwon GY: KillianJamieson diverticulum mimicking a suspicious thyroid nodule: Sonographic diagnosis. J Clin Ultrasound 37: 528-530, 2009.

18. Kang HC: A case of Zenker's diverticulum masquerading as a thyroid nodule. Korean J Med 67: 757-760, 2004.

19. Lixin J, Bing H, Zhigang W and Binghui Z: Sonographic diagnosis features of Zenker diverticulum. Eur J Radiol 80: e13-e19, 2011.

20. Huang YC, Chen JW and Chang CH: Is it really a thyroid nodule? Gastroenterology 145: 726-729, 2013.

21. Kim J, Kim YJ, Kim EK and Park CS: Incidentally found pharyngoesophageal diverticulum on ultrasonography. Yonsei Med J 43: 271-273, 2002.

22. Chea CH, Siow SL, Khor TH and Nik Azim NA: Killian-jamieson diverticulum: The rarer cervical esophageal diverticulum. Med J Malaysia 66: 73-74, 2011. 